\title{
Numerical Simulation of the Time Evolution of Small-Scale Irregularities in the F-Layer Ionospheric Plasma
}

\author{
O. V. Mingalev, G. I. Mingaleva, M. N. Melnik, and V. S. Mingalev \\ Kola Scientific Center of the Russian Academy of Sciences, Polar Geophysical Institute, Fersman street 14, 184209 Apatity, \\ Murmansk Region, Russia \\ Correspondence should be addressed to V. S. Mingalev, mingalev@pgia.ru
}

Received 15 February 2011; Revised 30 May 2011; Accepted 31 May 2011

Academic Editor: Lucilla Alfonsi

Copyright (๑) 2011 O. V. Mingalev et al. This is an open access article distributed under the Creative Commons Attribution License, which permits unrestricted use, distribution, and reproduction in any medium, provided the original work is properly cited.

Dynamics of magnetic field-aligned small-scale irregularities in the electron concentration, existing in the F-layer ionospheric plasma, is investigated with the help of a mathematical model. The plasma is assumed to be a rarefied compound consisting of electrons and positive ions and being in a strong, external magnetic field. In the applied model, kinetic processes in the plasma are simulated by using the Vlasov-Poisson system of equations. The system of equations is numerically solved applying a macroparticle method. The time evolution of a plasma irregularity, having initial cross-section dimension commensurable with a Debye length, is simulated during the period sufficient for the irregularity to decay completely. The results of simulation indicate that the smallscale irregularity, created initially in the F-region ionosphere, decays accomplishing periodic damped vibrations, with the process being collisionless.

\section{Introduction}

Occurrence of electron density irregularities is a natural phenomenon in the Earth's ionosphere. These irregularities have a wide range of spatial scales, ranging from a few Debye lengths to thousands of kilometers. The electron density increases and depletions inside irregularities can lie in the range from a few portions to some tens of percentages. The well-known equatorial anomaly is the example of large-scale irregularities in the ionospheric $\mathrm{F}$ layer. Another example is the main ionospheric trough observed at subauroral latitudes [1]. The high-latitude ionosphere can contain some types of large-scale irregularities, for example, the tongue of increased values of the electron density stretched over the polar cap from the dayside to night side, polar and auroral peaks of ionization, "patches" and "blobs" of ionization, and so on [2-5]. Middle-scale irregularities with dimensions of tens of kilometers are observed in the F-layer ionosphere, too $[6,7]$. It is known that small-scale irregularities, which can exist in the ionosphere, are predominately magnetic field aligned. The Earth's ionosphere can contain short-scale irregularities, having cross-section diameters of about hundreds of meters
[8-11]. Also, radio aurora or small-scale turbulence of the $\mathrm{E}$ layer is the well-known example of short-scale irregularities [12]. Meteor plasma trails are the example of irregularities in the ionosphere, too [13].

It is known that ionospheric irregularities may be originated not only by natural processes but also as a result of active experiments in the ionospheric plasma, in particular, as a result of release of various chemically active substances into the ionosphere. Moreover, ionospheric irregularities may be formed by high-power high-frequency radio waves, pumped into the ionosphere by ground-based ionospheric heaters. These waves cause a variety of physical processes in the ionospheric plasma. Some such processes result in the formation of both large-scale electron temperature and density irregularities and small-scale, geomagnetic field-aligned irregularities in the ionosphere. Mathematical modeling of the large-scale F-layer modification by powerful high frequency waves was performed in some studies, in particular, in the papers by Meltz and LeLevier [14], Perkins and Roble [15], Mantas et al. [16], Bernhardt and Duncan [17], Hansen et al. [18], Vas'kov et al. [19], and Mingaleva et al. [20, 21]. The parameters of the small-scale irregularities, formed in 
the F-layer ionosphere by powerful high frequency waves, were described in detail by Wong et al. [22]. According to their paper, diametrical sizes of these irregularities are several Debye lengths (no more that about 100), while the disturbances of the electron density in them can reach some tens of percentages. The formation of the small-scale irregularities in the F-layer ionosphere by powerful high frequency waves was considered and simulated in the study by Eliasson and Stenflo [23]. It can be noticed that such irregularities may be formed in the ionospheric plasma not only artificially but also by natural processes [22].

Just small-scale, geomagnetic field-aligned ionospheric irregularities are investigated in the present study utilizing a mathematical model developed recently in the Polar Geophysical Institute (PGI).

\section{Mathematical Model}

The ionospheric plasma at F-layer altitudes is supposed to be a rarefied compound consisting of electrons and positive ions in the presence of a strong, external, uniform magnetic field. The studied irregularities are assumed to be geomagnetic field-aligned, with their cross-sections being circular. The initial cross-section diameters of the irregularities are supposed to be commensurable with the Debye length. At F-layer levels, the mean free path of particles (electrons and ions) between successive collisions is much more than the cross-section diameters of the considered irregularities. Therefore, the plasma is assumed to be collisionless. Kinetic processes in such plasma are described by the Vlasov-Poisson system of equations which has been considered, for example, in the studies by Hockney and Eastwood [24], and Birdsall and Langdon [25]. This system may be written as follows:

$$
\frac{\partial f_{a}}{\partial t}+\left(\mathbf{v}, \frac{\partial f_{a}}{\partial \mathbf{x}}\right)+\frac{q_{a}}{m_{a}}\left(\mathbf{E}+\left[\mathbf{v} \times \mathbf{B}_{0}\right], \frac{\partial f_{a}}{\partial \mathbf{v}}\right)=0, \quad a=i, e,
$$

$$
\begin{gathered}
\Delta \varphi(\mathbf{x}, t)=-\frac{1}{\varepsilon_{0}} \rho(\mathbf{x}, t), \\
\mathbf{E}(\mathbf{x}, t)=-\nabla \varphi(\mathbf{x}, t), \quad \rho(\mathbf{x}, t)=e_{0}\left(n_{i}-n_{e}\right), \\
n_{a}(\mathbf{x}, t)=\int f_{a}(t, \mathbf{x}, \mathbf{v}) d \mathbf{v}
\end{gathered}
$$

where $f_{a}(t, \mathbf{x}, \mathbf{v}), n_{a}(\mathbf{x}, t), m_{a}$, and $q_{a}$ are, respectively, the distribution function, concentration, mass, and charge of particles of type $a, \mathbf{x}$ is the space coordinate vector, $\mathbf{v}$ is the velocity, $\mathbf{B}_{0}$ is the external magnetic field, $\mathbf{E}$ is the selfconsistent electric field, $\varphi(\mathbf{x}, t)$ is the electric field potential, $\rho(\mathbf{x}, t)$ is the electric charge density, $\varepsilon_{0}$ is the dielectric constant of free space, and $e_{0}$ is the proton charge. The Vlasov equation, (1), describes the evolution of the distribution functions of charged particles and the Poisson equation, (2), describes the self-consistent electric field.

As pointed out previously, the investigated irregularities are geomagnetic field-aligned. Their longitudinal sizes are much more than the cross-section diameters. Gradients of the plasma parameters in the longitudinal direction are much less than those in a plane perpendicular to a magnetic field in the vicinity of the irregularity. Therefore, plasma parameters in the vicinity of the irregularity may be considered as independent on the longitudinal coordinate. This simplification allows us to consider a two-dimensional flow of plasma in a plane perpendicular to a magnetic field line.

Not long ago, in the PGI, the two-dimensional mathematical model has been developed which is intended to simulate dynamics of the near-earth rarefied plasma [26]. In this model, the full implicit variant of the macroparticle method is applied for joint numerical solving of the Vlasov equations, (1), and the Poisson equation, (2), with the real charge-mass ratio for electrons having been used. The Poisson equation, (2), is numerically solved using a finite-difference method having the forth order of accuracy. This model has been utilized for numerical simulation of the behavior of smallscale irregularities of the electron density which can exist in the magnetospheric plasma [26]. In the present study, this model is utilized to investigate the time evolution of smallscale irregularities in the F-layer ionospheric plasma.

In this work, the simulation region lays in the plane perpendicular to the magnetic field line. The simulation region is a square and its side length is equal to 96 Debye lengths of plasma. The dimension of the simulation region is consistent with recommendations of the applied macroparticle method using a discrete Vlasov-Poisson system of equations. According to these recommendations, for the adequate representation of the real plasma, the dimension of the simulation region should be no less than 60-100 Debye length of the plasma $[24,25]$.

The grid width is equal to one eighth of the Debye length of the plasma. The quantity of the grid cells is $768 \times 768$. The time step for the electric field is equal to one hundredth of an equilibrium period of Langmuir oscillations of electrons. The periodical boundary conditions for the distribution func-tions and electric field are utilized in this work. In the model calculations, the number of macro-particles per species is $72 \cdot 2^{20} \approx 75.5 \cdot 10^{6}$; therefore, the average number of macro-particles in the Debye cell for the model plasma is equal to $2^{13}$.

The temporal evolution of the small-scale irregularity, created initially in the F-layer ionospheric plasma, is numerically studied during a period sufficient for the irregularity to decay completely. The $2 \mathrm{D} 2 \mathrm{~V}$ variant of the mathematical self-consistent model of dynamics of the near-earth rarefied plasma is utilized. More specific details of the utilized mathematical model and peculiarities of the applied numerical method may be found in the study of Mingalev et al. [26].

\section{Presentation and Discussion of Results}

The utilized mathematical model can describe the behavior of the near-earth plasma under various conditions. The results of calculations to be presented in this paper were obtained using the input parameters of the model typical for the nocturnal ionospheric plasma at the level of $300 \mathrm{~km}$. In particular, the value of the nondisturbed electron concentration (equal to the positive ion concentration) is $10^{11} \mathrm{~m}^{-3}$. 
The electron and ion temperatures are supposed to be equal to $1213 \mathrm{~K}$ and $930 \mathrm{~K}$, respectively. The bulk flow velocities of electrons and positive ions are assumed to be zero. The value of the magnetic field, $B_{0}$, is $4.4 \cdot 10^{-5} \mathrm{~T}$.

The above pointed out values yield the following quantities of some physically significant parameters. The electron thermal velocity, $V_{T e}$, is equal to $135.6 \mathrm{~km} / \mathrm{s}$, the equilibrium plasma frequency, $\omega_{p e}^{0}$, is $1.78 \cdot 10^{7} \mathrm{~s}^{-1}$. The Debye length of the plasma, $\lambda_{D e}^{0}$, defined as $\lambda_{D e}^{0}=V_{T e} / \omega_{p e}^{0}$, is equal to $7.6 \cdot 10^{-3} \mathrm{~m}$; the electron gyro radius, $R_{c e}$, is $17.5 \cdot 10^{-3} \mathrm{~m}$. The equilibrium period of Langmuir oscillations of electrons, $T_{p e}$, is $3.52 \cdot 10^{-7} \mathrm{~s}$. The period of cyclotron oscillations of electrons, $T_{c e}$, is equal to $8.12 \cdot 10^{-7} \mathrm{~s}$, that is, approximately a factor of 2.3 larger than the equilibrium period of Langmuir oscillations of electrons $\left(T_{c e} \approx 2.3 \cdot T_{p e}\right)$. The collision mean free path of charged particles is about $50 \mathrm{~m}$. The mean free time of electron between successive collisions with other particles, $T_{\text {freepath }}$, is larger than the equilibrium period of Langmuir oscillations of electrons by a factor of about 1047 $\left(T_{\text {freepath }} \approx 1047 T_{p e}\right)$.

Taking the input parameters of the mathematical model typical for the nocturnal ionosphere at the level of $300 \mathrm{~km}$, we have calculated the time evolution of the distribution functions of charged particles as well as self-consisting electric field for two distinct on principle situations. In these situations, the initial distributions of electric charge density have been different. The first situation corresponds to homogeneous spatial distributions of the electron and positive ion concentrations at the initial moment inside the simulation region, with the plasma being electrically neutral and the electric charge density being equal to zero.

The second situation corresponds to homogeneous spatial distribution of the positive ion concentration only. The spatial distribution of the electron concentration, at the initial moment, contains a circular irregularity at the center of the simulation region. Inside the irregularity, the electric neutrality of the plasma is broken whereas, beyond it, the plasma is electrically neutral at the initial moment.

Simulation results, obtained for the first situation when the process started from the completely electrically neutral state, indicate that the spatial distributions of the electron and positive ion concentrations tend to retain a homogeneity and electrical neutrality of the plasma. However, short-scale nonregular fluctuations of the calculated parameters of the plasma arise near their initial values. In particular, the electric charge density and electric field fluctuate, with amplitudes of the fluctuations being very little. We calculate two orthogonal components of the electric field, $E_{x}$ and $E_{y}$, lying in the plane perpendicular to the magnetic field. It turns out that the maximal amplitudes of the electric field component fluctuations do not exceed a value of $10^{-5} \mathrm{~V} / \mathrm{m}$ at all grid cells of the simulation region.

It is of interest to consider a decrease of electron concentration, $n_{e}$, relatively to the initial value of the nondisturbed electron concentration (equal to the positive ion concentration), $n_{0}$, that is, the ratio $\left(n_{0}-n_{e}\right) / n_{0}$, which will be referred to as a relative decrease of the electron concentration. The results of calculations indicate that the maximal amplitude of

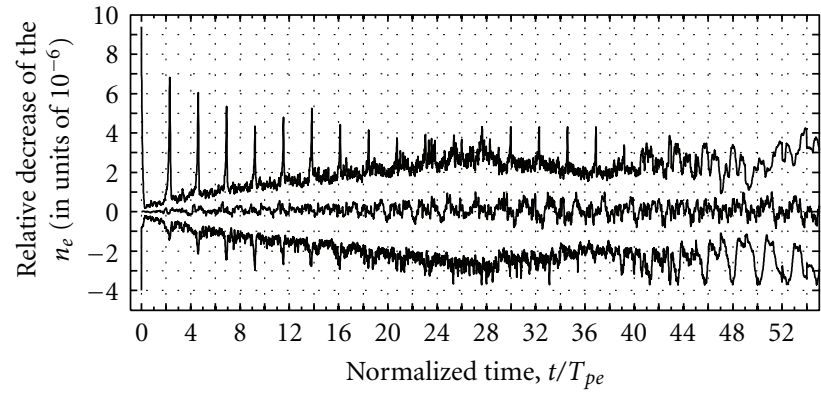

FIGURE 1: The time variations of maximal (top curve) and minimal (bottom curve) values of the relative decrease of the electron concentration, computed at all grid cells of the simulation region. Also, the time variation of the relative decrease of the electron concentration, calculated at the center of the simulation region (middle curve). The results were obtained for the first situation with the start from the completely electrically neutral state. The values are given in units of $10^{-6}$. The normalized time, $t / T_{p e}$, that is, the time in units of the equilibrium period of Langmuir oscillations of electrons, $T_{p e}$, is shown on the abscissa.

this quantity does not exceed a value of $10^{-5}$ at all grid cells of the simulation region (Figure 1). At one separate point of the simulation region, the calculated parameters of the plasma fluctuate near their initial values (Figure 1, middle curve).

One of the physically significant parameters of the plasma, filling in a volume $X$, is a potential energy of the plasma

$$
W_{\text {pot }}(t)=\frac{1}{2} \int_{X} \rho \cdot \varphi d x,
$$

where $\rho$ and $\varphi$ are the same quantities as in (2). Let $W_{\text {kin }}^{0}$ be the initial kinetic energy of electrons filling in the volume $X$. A normalized potential energy of the plasma may be defined as $W_{\text {pot }}(t) / W_{\text {kin }}^{0}$.

Results of simulation, obtained for the first situation with the start from the completely electrically neutral state, indicate that the normalized potential energy of the plasma fluctuates (Figure 2). It is seen that the amplitudes of these fluctuations for the first situation did not exceed a value of $13 \cdot 10^{-11}$ (Figure 2). The period of plasma parameters fluctuations of shortest duration is about $(0.1-0.2) \cdot T_{p e}$. Besides, fluctuations with other periods may take place in the simulation results, in particular, with the equilibrium period of Langmuir oscillations of electrons, $T_{p e}$, and period of cyclotron oscillations of electrons, $T_{c e}$, (Figures 1 and 2).

The presence of the short-scale nonregular fluctuations of the calculated parameters of the plasma, referred to as a discrete noise, is due to the specific character of the applied macroparticle method, with the fluctuation amplitudes being conditioned by the number of macro-particles used in the calculations. It can be noticed that, in the present calculations, we use the discrete 2D2V Maxwell distribution simulated by $2^{11}$ macro-particles with 16 levels of energy and 128 points for azimuth angle of velocity, which were uniformly located in space in a square having the side length of $0.5 \cdot \lambda_{D e}^{0}$. 


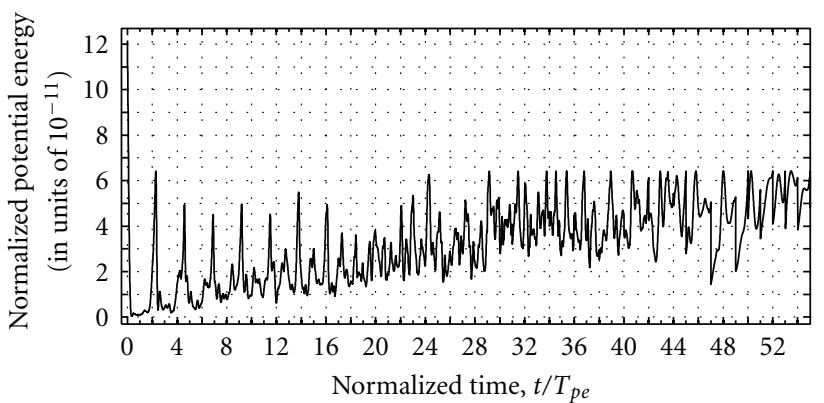

FIgURE 2: The time variation of the normalized potential energy of the plasma filling up all simulation region, $W_{\text {pot }}(t) / W_{\text {kin }}^{0}$. The results were obtained for the first situation with the start from the completely electrically neutral state. The values are given in units of $10^{-11}$. The normalized time, $t / T_{p e}$, that is, the time in units of the equilibrium period of Langmuir oscillations of electrons, $T_{p e}$, is shown on the abscissa.

It should be emphasized that the amplitudes of the fluctuations, obtained for the first situation when process started from the completely electrically neutral state, characterize the accuracy of the applied numerical method that cannot be exceeded in following calculations. The determination of this accuracy is one of the goals of performing calculations for the first situation corresponding to homogeneous spatial distributions of electrons and positive ions inside the simulation region at the initial moment.

Let us consider the results of simulation, obtained for the second situation when, at the initial moment, the spatial distribution of the electron concentration contains a circular irregularity at the center of the simulation region, with the spatial distribution of the positive ion concentration being homogeneous in all simulation region. Calculations were made for the case in which the initially created irregularity has the cross-section diameter of $12 \lambda_{D e}^{0}$, where $\lambda_{D e}^{0}$ is the Debye length of the plasma. Inside this irregularity, a part of electrons traveled from the internal circle, having the diameter of $6 \lambda_{D e}^{0}$, into the external ring, surrounding the internal circle. Calculations were performed for the case in which the relative decrease of the electron concentration, $\left(n_{0}-n_{e}\right) / n_{0}$, is equal to 0.2 in the internal circle at the initial moment. In other words, 20 percent of electrons were displaced from the center of the irregularity to its periphery. As a consequence of this displacement, an excess of positive charge appeared in the internal circle of the irregularity, while an excess of negative charge appeared in the ring surrounding it. The configuration of the initial irregularity in the plane perpendicular to the magnetic field is presented in Figure 3(a). It can be noticed that the shape, dimensions, and disturbance of the electron density of the considered irregularity correspond to the parameters of the small-scale irregularities, described in the study by Wong et al. [22]

The time evolution of the initially created irregularity was numerically simulated using the mathematical model described above. Simulation results indicate that, after initial moment, the spatial distribution of the electron concentration changes essentially while the positive ion concentration is retained practically invariable. It turns out that the initially created irregularity vanishes completely during a short period, with the plasma becoming electrically neutral in all simulation region at the moment near to the equilibrium period of Langmuir oscillations of the electrons $\left(t \approx T_{p e}\right)$. Figure 3(b) illustrates this condition. Further calculations indicate that the changes in the electron concentration are continued and, after a short period, the irregularity arises again to a moment of about $(2.2-2.3) T_{p e}$. This fact is illustrated by Figure 3(c). It can be seen that the recovered irregularity almost completely coincides with the initial one presented in Figure 3(a). Later, the cycle of vanishing and recovering of the irregularity is repeated again and again (see Figures 3(d) and 3(e)). Thus, the time evolution of the initially created irregularity is accompanied by periodic vibrations. The period of these vibrations is approximately a factor of 2.3 larger than the equilibrium period of Langmuir oscillations of electrons, with the former period coinciding with the period of cyclotron oscillations of electrons, $T_{c e}$. In the course of time, the amplitudes of the vibrations of the electron concentration and electric field decrease smoothly, with the irregularity decaying little by little.

It is of interest to note that, in the process of evolution, additional almost symmetrical alternate rings with an excess of charge of different sign begin to appear around the initial irregularity situated in the center of the simulation region. Such rings are seen in Figure 3(e) where the spatial distribution of the relative decrease of the electron concentration, obtained after approximately 2 oscillation periods, is shown. In the course of time, these additional rings begin to fill up all simulation region, with the irregularity center remaining immovable. This condition can be seen in Figure 3(f), illustrating the spatial distribution of the relative decrease of the electron concentration, calculated after approximately 6 oscillation periods.

Simulation results, obtained for the second situation when the temporal evolution of the initially created irregularity was studied, indicate that the calculated parameters fluctuate at separate points of the simulation region. Examples of fluctuating parameters of the plasma are presented in Figure 4. It is seen from the bottom panel of Figure 4 that the amplitudes of the electric field fluctuations, obtained for the second situation when the initially created irregularity was studied, are much more than those, obtained for the first situation when the process started from the completely electrically neutral state (Figure 4(a)). In the first situation, they did not exceed a value of $10^{-5} \mathrm{~V} / \mathrm{m}$ while, in the second situation, they can exceed a value of $0.2 \mathrm{~V} / \mathrm{m}$ (Figure 4 ). In the second situation, it is easy to see that the electric field fluctuations possess of two main periods, namely, the period of Langmuir oscillation of electrons and period of cyclotron oscillations of electrons (Figure 4(b)).

Results of simulation, obtained for the second situation, indicate that the normalized potential energy of the plasma can fluctuate. From Figure 5, it is seen that the normalized potential energy of the plasma, $W_{\text {pot }}(t) / W_{\text {kin }}^{0}$, demonstrates periodic damped vibrations. The period of the vibrations, having maximal amplitudes, is close to the period of cyclotron oscillations of electrons, which is approximately 


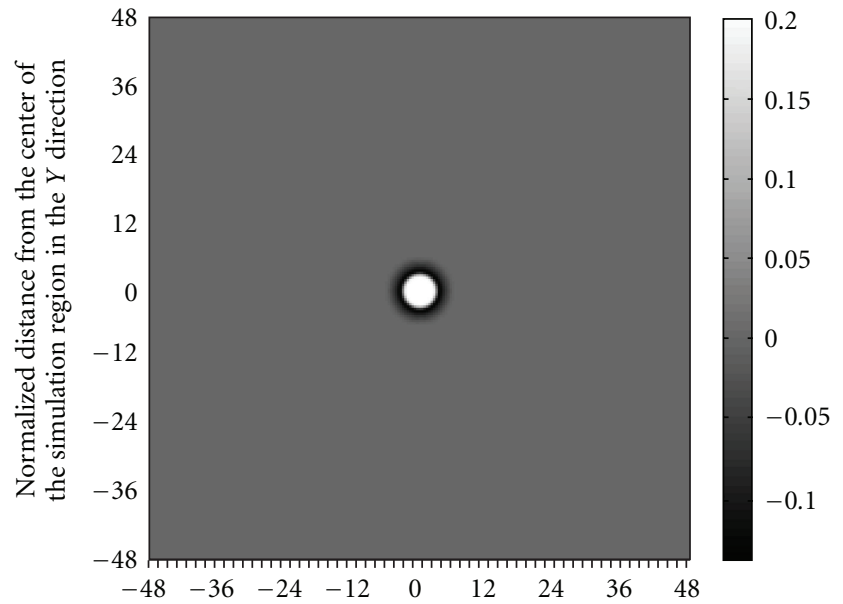

(a)

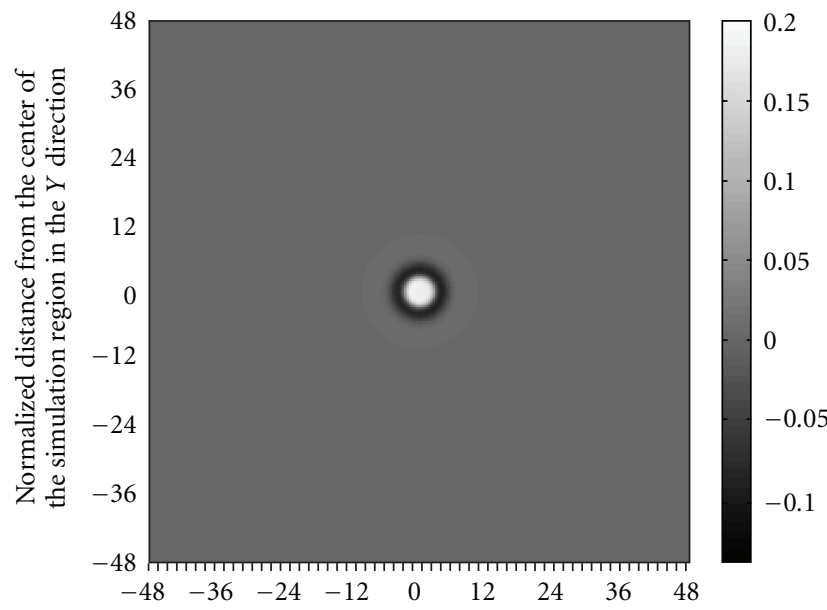

(c)

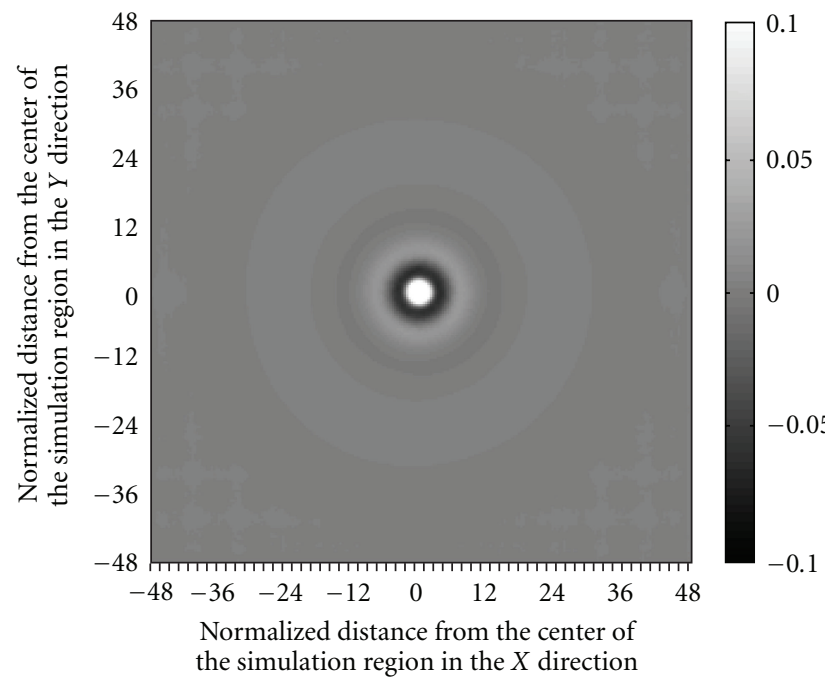

(e)

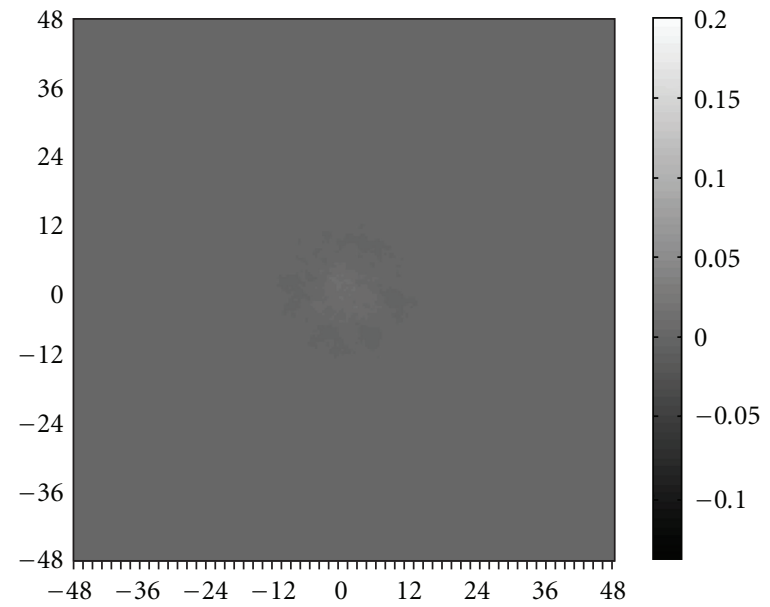

(b)

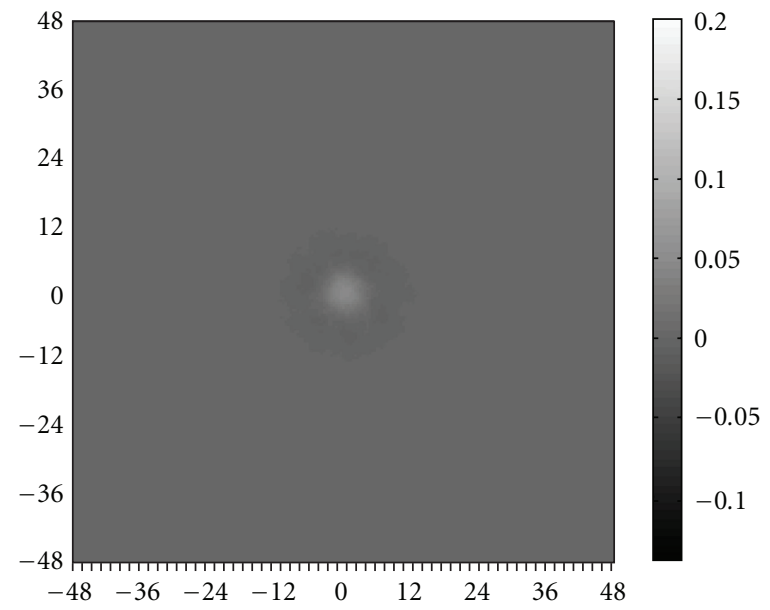

(d)

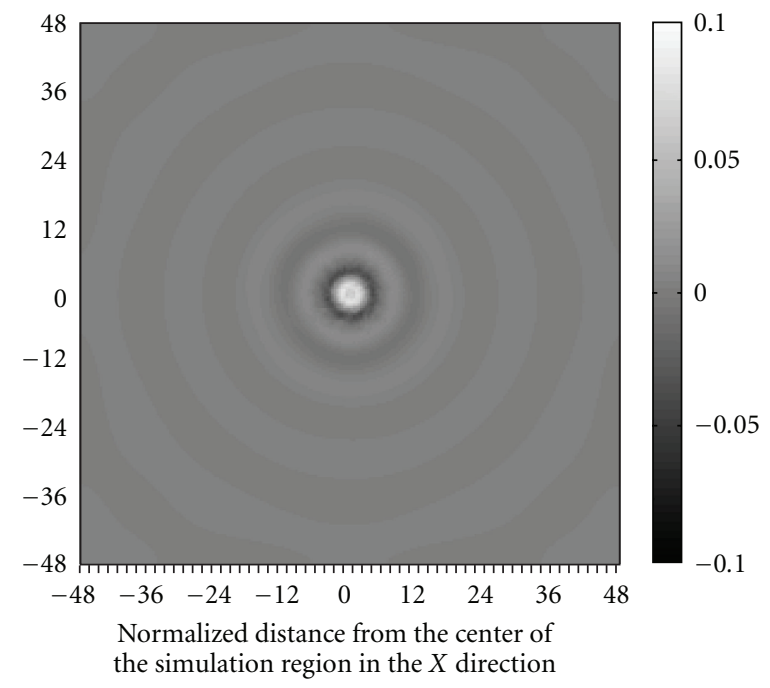

(f)

FIgURE 3: The calculated spatial distributions of the relative decrease of the electron concentration, $\left(n_{0}-n_{e}\right) / n_{0}$ in the plane perpendicular to the magnetic field. The normalized distances, $\mathbf{X} / \lambda_{D_{e}}^{0}$ and $\mathbf{Y} / \lambda_{D_{e}}^{0}$, that is, the distances in units of the Debye length, $\lambda_{D_{e}}^{0}$, from the central point of the simulation region are shown on the horizontal $(\mathbf{X})$ and vertical $(\mathbf{Y})$ axes. The results, obtained for the second situation when the temporal evolution of the initially created irregularity was studied, are given for the following moments: (a) $t=0$, (b) $t=T_{p e}$, (c) $t=2.2 \cdot T_{p e}$, (d) $t=3 \cdot T_{p e}$, (e) $t=4.4 \cdot T_{p e}$, and (f) $t=14.09 \cdot T_{p e}$. 


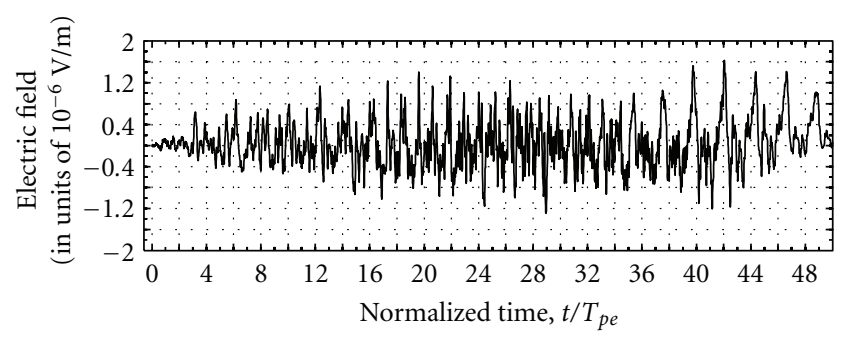

(a)

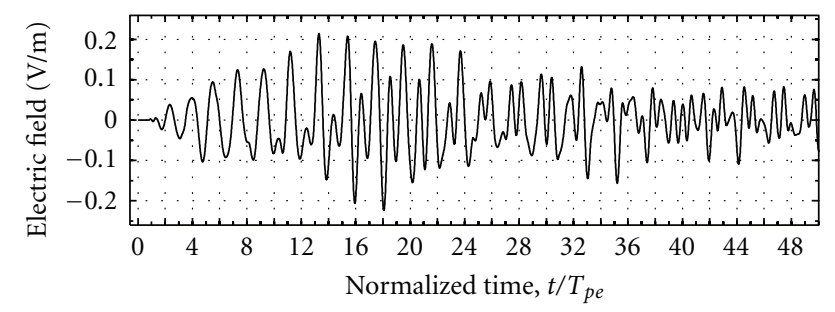

(b)

Figure 4: The time variations of one component of the electric field, namely, $E_{x}$, lying in the plane perpendicular to the magnetic field, calculated at the center of the simulation region for the first situation with the start from the completely electrically neutral state (a), also $E_{x}$, calculated at the point, displaced from the center of the simulation region in the $\mathbf{X}$ direction for a distance of sixteen Debye lengths $\left(16 \cdot \lambda_{D_{e}}^{0}\right)$, for the second situation when the initially created irregularity was studied (b). The electric field components are given in units of $10^{-6} \mathrm{~V} / \mathrm{m}$ in (a) and in $\mathrm{V} / \mathrm{m}$ in the (b). The normalized time, $t / T_{p e}$, that is, the time in units of the equilibrium period of Langmuir oscillations of electrons, $T_{p e}$, is shown on the abscissa.

a factor of 2.3 larger than the equilibrium period of Langmuir oscillations of electrons. The amplitude of these vibrations decreases from the initial value of about 0.08 to a quantity, approximately a factor of 10 less than the initial value, with the achieved quantity remaining almost invariable in further calculations (Figure 5). The time interval of about $35 T_{p e}$ (approximately 15 vibration periods of the irregularity) is sufficient for the amplitude of the normalized potential energy of the plasma to become almost invariable. Results of simulations indicate that, during this time interval, the considered irregularity lost almost completely its initial structure, was diffused, and decayed. It should be emphasized that this time interval is much less than the mean free time of electron between successive collisions with other particles. Consequently, the process of decaying the initially created irregularity is really collisionless, with the application of the Vlasov-Poisson system of equations for the description of this process having been justified.

As was noted earlier, the applied mathematical model has been utilized for numerical simulation of the behavior of small-scale irregularities of the electron density which can exist in the magnetospheric plasma [26]. In spite of differences of plasma parameters in the magnetosphere and ionosphere, the behavior of small-scale irregularities turns out to be similar. Magnetospheric small-scale irregularities were found to decay accomplishing periodic damped

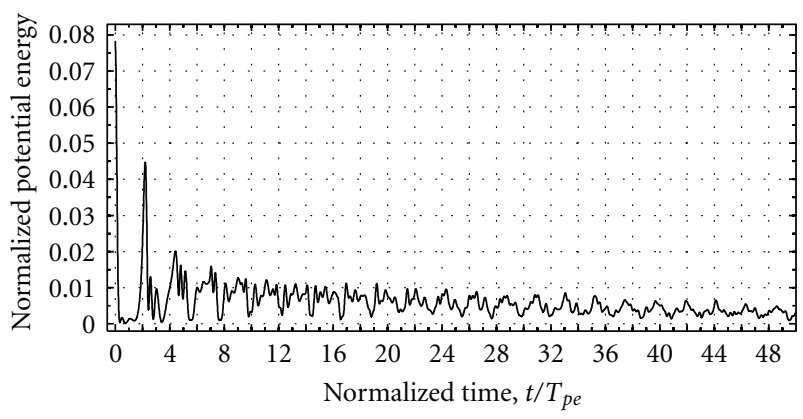

FIGURE 5: The time variation of the normalized potential energy of the plasma filling up all simulation region, $W_{\text {pot }}(t) / W_{\text {kin }}^{0}$. The results were obtained for the second situation when the initially created irregularity was studied. The normalized time, $t / T_{p e}$, that is, the time in units of the equilibrium period of Langmuir oscillations of electrons, $T_{p e}$, is shown on the abscissa.

vibrations. The period of the vibrations with maximal amplitudes turned out to be a factor of approximately 1.3-1.4 larger than the equilibrium period of Langmuir oscillations of electrons. The former period proves to be equal to the period of cyclotron oscillations of electrons. The time interval of approximately 20 Langmuir oscillation periods of electrons (about 15 vibration periods of the irregularity) was a characteristic time of existence of the initially created irregularity in the magnetospheric plasma at the level of about $3000 \mathrm{~km} \mathrm{[26].} \mathrm{It} \mathrm{can} \mathrm{be} \mathrm{seen} \mathrm{that,} \mathrm{in} \mathrm{essence,} \mathrm{the}$ results, presented in this paper, are similar to the results, obtained in [26].

\section{Summary and Concluding Remarks}

In this paper, the time evolution of the small-scale irregularities in the F-layer ionospheric plasma was numerically simulated using the two-dimensional mathematical model, developed recently in the PGI. The model is based on numerical solving the Vlasov-Poisson system of equations, with the Vlasov equations describing the distributions functions of charged particles and the Poisson equation governing the self-consistent electric field. The full implicit variant of the macro-particle method is applied for numerical solving of the system of equations, with the real charge-mass ratio for electrons having been used.

The investigated irregularities are supposed to be geomagnetic field-aligned, with their cross-section being circular. The cross-section diameters of the irregularities are much less than their longitudinal dimensions. Gradients of the plasma parameters in a plane perpendicular to a magnetic field are much more than those in the longitudinal direction in the vicinity of the irregularity. Consequently, the latter gradient may be omitted and the flow of plasma may be considered as two-dimensional in the plane perpendicular to the magnetic field.

Calculations were made of the time evolution of the distribution functions of charged particles as well as the self-consisting electric field for conditions typical for the nocturnal ionospheric plasma at the level of $300 \mathrm{~km}$. Firstly, 
to determine the accuracy of the mathematical model, calculations were performed for the situation corresponding to homogeneous spatial distributions of electrons and positive ions inside the simulation region at the initial moment, that is, when the process started from the completely electrically neutral state. As a consequence, the amplitudes of the socalled discrete noise of the numerical model were determined.

Secondly, the temporal evolution of the initially created irregularity was numerically simulated which has the parameters typical for small-scale irregularities in the F-layer ionospheric plasma. Simulation results indicated that, in the course of time, the irregularity decays accomplishing periodic damped vibrations.

The irregularity vanishes and recovers periodically, with its parameters fluctuating. In particular, the normalized potential energy of the plasma demonstrates periodic damped vibrations. The period of the vibrations with the maximal amplitudes is close to the period of cyclotron oscillations of electrons which is approximately a factor of 2.3 larger than the equilibrium period of Langmuir oscillations of electrons. During the process of evolution, around the initial irregularity, additional almost symmetrical alternate rings with an excess of charge of different sign began to appear. In the course of time, these additional rings filled up all simulation region.

The time interval of about 15 vibration periods of the irregularity is sufficient for the irregularity to lose almost completely its initial structure, to be diffused, and to decay. This time interval is much less than the mean free time of electron between successive collisions with other particles. Therefore, the process of destroying the initially created irregularity is substantially collisionless.

\section{Acknowledgments}

This study was partially supported by the Division of Physical Sciences of the Russian Academy of Sciences through the program "Plasma processes in the solar system" and by the RFBR Grant no. 10-01-00451.

\section{References}

[1] R. J. Moffett and S. Quegan, "The mid-latitude trough in the electron concentration of the ionospheric F-layer: a review of observations and modelling," Journal of Atmospheric and Terrestrial Physics, vol. 45, no. 5, pp. 315-343, 1983.

[2] J. Buchau, B. W. Reinisch, E. J. Weber, and J. G. Moore, "Structure and dynamics of the winter polar cap F region," Radio Science, vol. 18, no. 6, pp. 995-1010, 1983.

[3] R. W. Robinson, R. T. Tsunoda, J. F. Vickrey, and L. Guerin, "Sources of $\mathrm{F}$ region ionization enhancement in the nighttime auroral zone," Journal of Geophysical Research, vol. 90, pp. 7533-7546, 1985.

[4] R. T. Tsunoda, "High-latitude F region irregularities: a review and synthesis," Reviews of Geophysics, vol. 26, pp. 719-760, 1988.

[5] A. S. Besprozvannaya, G. A. Zherebtsov, O. M. Pirog, and T. I. Shchuka, "Dynamics of electron density in the auroral zone during the magnetospheric substorm on December 22, 1982," Geomagnetism and Aeronomy, vol. 28, no. 1, pp. 66-70, 1988.

[6] D. B. Muldrew and J. F. Vickrey, "High-latitude F region irregularities observed simultaneously with ISIS1 and Chatanika radar," Journal of Geophysical Research, vol. 87, no. A10, pp. 8263-8272, 1982.

[7] S. Basu, E. Mac Kenzie, S. Basu et al., "Simultaneous density and electric field fluctuation spectra associated with velocity shears in the auroral oval," Journal of Geophysical Research, vol. 93, no. A1, pp. 115-136, 1988.

[8] E. J. Fremouw, C. L. Rino, R. C. Livingston, and M. C. Cousins, "A persistent subauroral scintillations enhancement observed in Alaska," Geophysical Research Letters, vol. 4, pp. 539-542, 1977.

[9] E. Martin and J. Aarons, "F layer scintillations and the aurora," Journal of Geophysical Research, vol. 82, pp. 2717-2722, 1977.

[10] L. Kersley, C. D. Russell, and S. E. Pryse, "Scintillation and EISCAT investigations of gradient-drift irregularities in the high latitude ionosphere," Journal of Atmospheric and Terrestrial Physics, vol. 51, no. 4, pp. 241-247, 1989.

[11] S. E. Pryse, L. Kersley, and C. D. Russell, "Scintillation near the F layer trough over northern Europe," Radio Science, vol. 26, no. 4, pp. 1105-1114, 1991.

[12] R. A. Greenwald, "Diffuse radar aurora and the gradient drift instability," Journal of Geophysical Research, vol. 79, pp. 48074810, 1974.

[13] Y. S. Dimant, M. M. Oppenheim, and G. M. Milikh, "Meteor plasma trails: effects of external electric field," Annales Geophysicae, vol. 27, no. 1, pp. 279-296, 2009.

[14] G. Meltz and R. E. LeLevier, "Heating the F-region by deviative absorption of radio waves," Journal of Geophysical Research, vol. 75, pp. 6406-6416, 1970.

[15] F. W. Perkins and R. G. Roble, "Ionospheric heating by radio waves: predictions for Arecibo and the satellite power station," Journal of Geophysical Research, vol. 83, no. 4, pp. 1611-1624, 1978.

[16] G. P. Mantas, H. C. Carlson, and C. H. La Hoz, "Thermal response of F-region ionosphere in artificial modification experiments by HF radio waves," Journal of Geophysical Research, vol. 86, no. A2, pp. 561-574, 1981.

[17] P. A. Bernhardt and L. M. Duncan, "The feedback-diffraction theory of ionospheric heating," Journal of Atmospheric and Terrestrial Physics, vol. 44, no. 12, pp. 1061-1074, 1982.

[18] J. D. Hansen, G. J. Morales, and J. E. Maggs, "Daytime saturation of thermal cavitons," Journal of Geophysical Research, vol. 94, no. A6, pp. 6833-6840, 1989.

[19] V. V. Vas'kov, Y. S. Dimant, and N. A. Ryabova, "Magnetospheric plasma thermal perturbations induced by resonant heating of the ionospheric F-region by high-power radio wave," Advances in Space Research, vol. 13, no. 10, pp. 25-33, 1993.

[20] G. I. Mingaleva, V. S. Mingalev, and I. V. Mingalev, "Simulation study of the high-latitude F-layer modification by powerful HF waves with different frequencies for autumn conditions," Annales Geophysicae, vol. 21, no. 8, pp. 1827-1838, 2003.

[21] G. I. Mingaleva, V. S. Mingalev, and I. V. Mingalev, "Model simulation of the large-scale high-latitude F-layer modification by powerful HF waves with different modulation," Journal of Atmospheric and Solar-Terrestrial Physics, vol. 71, no. 5, pp. 559-568, 2009.

[22] A. Y. Wong, J. Santoru, C. Darrow, L. Wang, and J. G. Roederer, "Ionospheric cavitons and related nonlinear phenomena," Radio Science, vol. 18, no. 6, pp. 815-830, 1983. 
[23] B. Eliasson and L. Stenflo, "Full-scale simulation study of the initial stage of ionospheric turbulence," Journal of Geophysical Research A, vol. 113, no. 2, Article ID A02305, 2008.

[24] R. W. Hockney and J. W. Eastwood, Computer Simulation Using Particles, McGraw-Hill, New York, NY, USA, 1981.

[25] C. K. Birdsall and A. B. Langdon, Plasma Physics via Computer Simulation, McGraw-Hill, New York, NY, USA, 1985.

[26] O. V. Mingalev, I. V. Mingalev, and V. S. Mingalev, "Twodimensional numerical simulation of dynamics of small-scale irregularities in the near-Earth plasma," Cosmic Research, vol. 44, no. 5, pp. 398-408, 2006. 

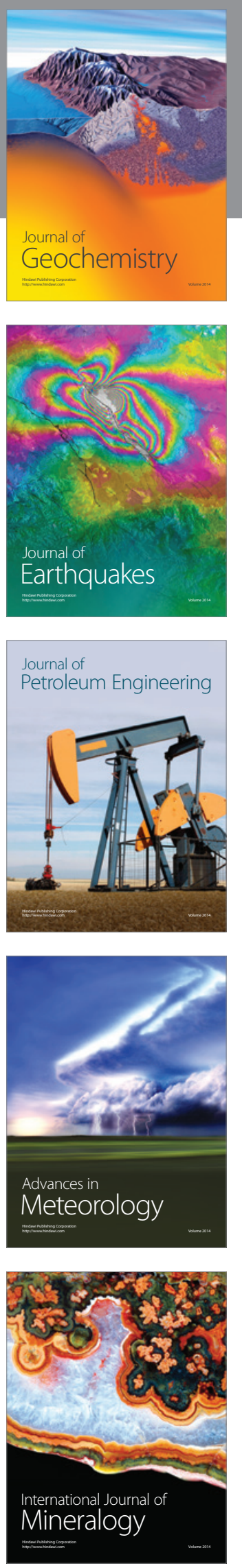
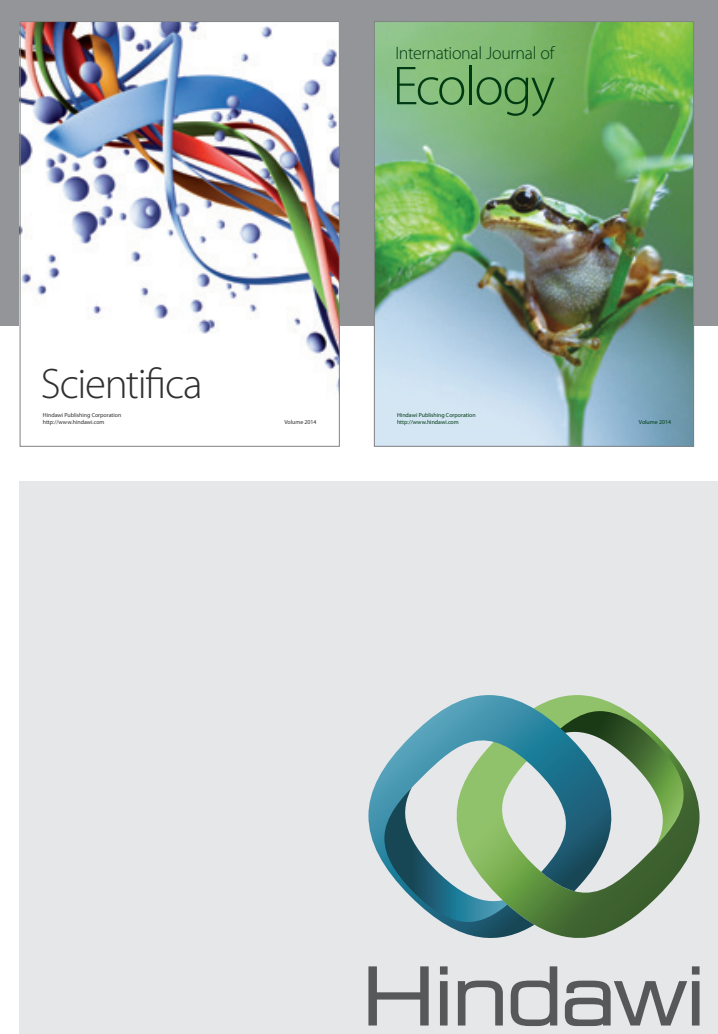

Submit your manuscripts at http://www.hindawi.com
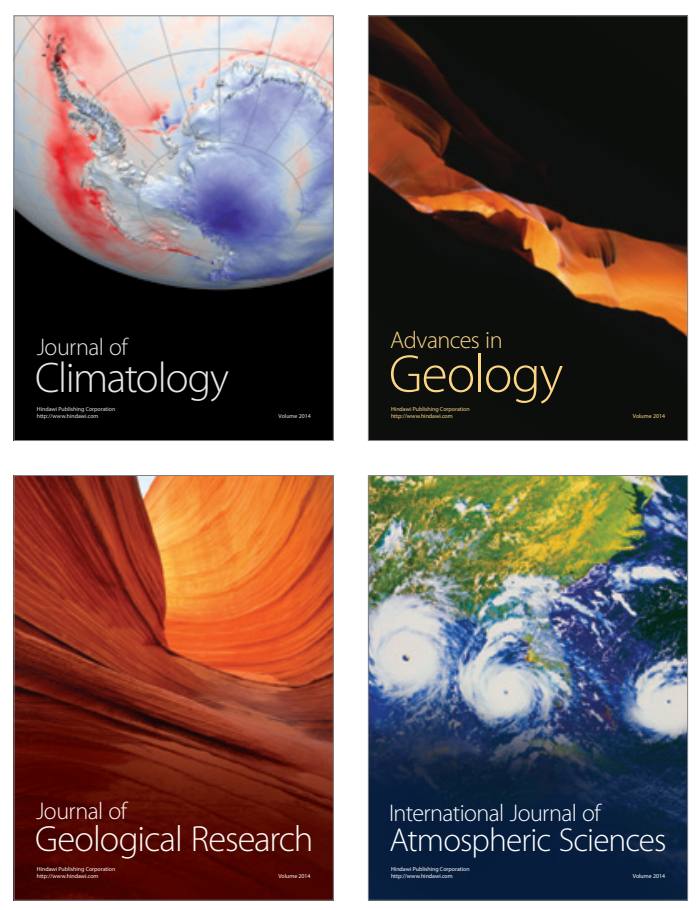
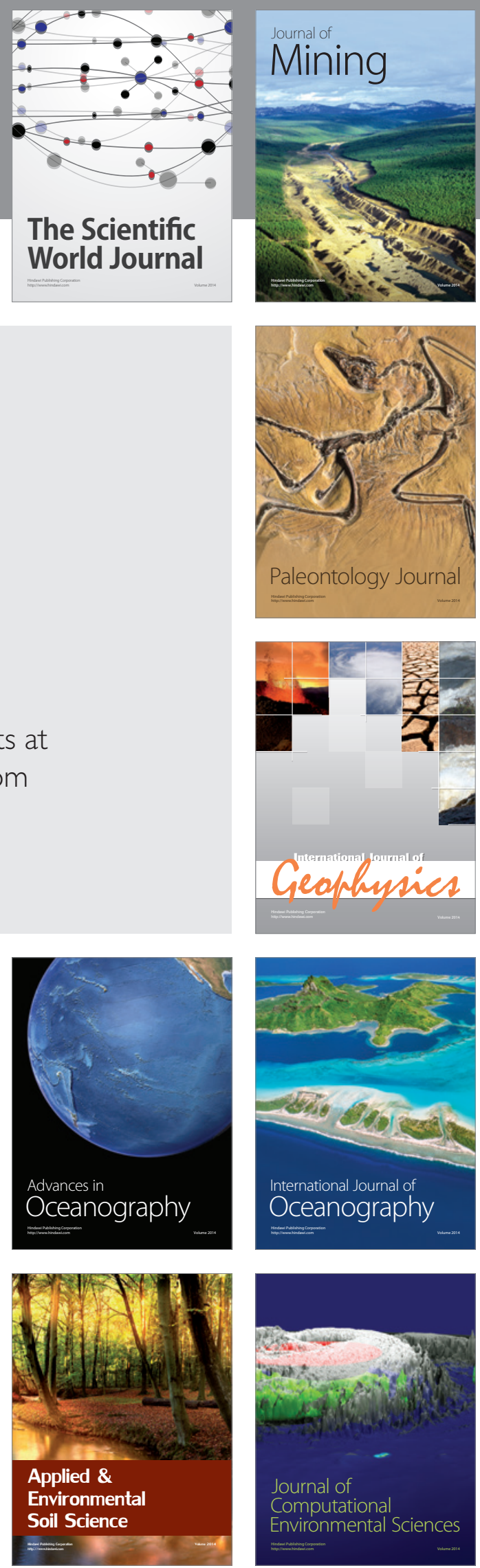